\title{
Interview with Edward A. Bender
}

\section{Toufik Mansour}

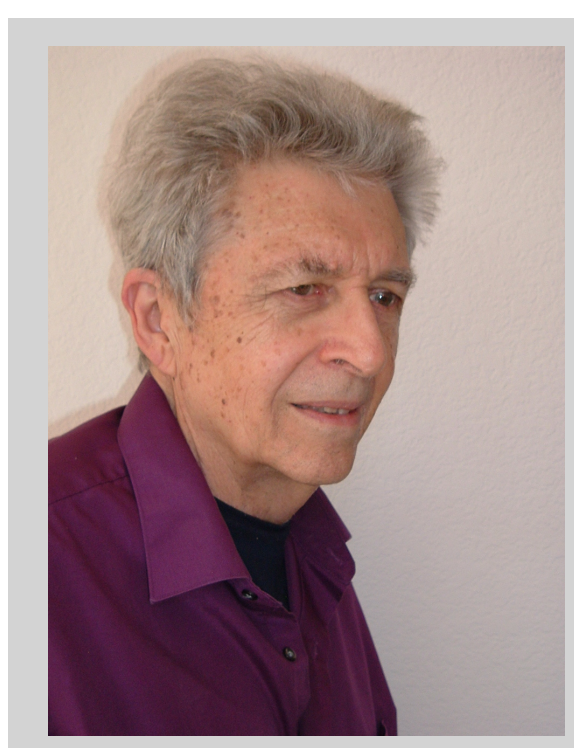

Photo by Arlene Bender
Edward Bender completed his undergraduate studies at the California Institute of Technology in 1963 and obtained a Ph.D. there in 1966, under the direction of Olga Taussky-Todd. His post-doctoral position 1966-1968 was at Harvard University. Between 1968-1974, he was full-time at the Institute for Defense Analyses/Communication Research Division in Princeton; now called the Center for Communications Research (CCR). Since 1974, he was a Professor of Mathematics in the Department of Mathematics at UC San Diego (UCSD) except for a year off to help set up a branch of CCR in La Jolla. Since 2008 he is Professor Emeritus. He is interested in enumeration and asymptotics in combinatorics, graph theory, and population biology. In these areas, he wrote several textbooks: An Introduction to Mathematical Modeling; Mathematical Methods in Artificial Intelligence; Foundations of Applied Combinatorics; Mathematics for Algorithms and Systems Analysis; A Short

Course in Discrete Mathematics; Foundations of Combinatorics with Applications; Lists, Decisions and Graphs - With an Introduction to Probability. The combinatorial texts were written jointly with S. Gill Williamson.

Mansour: Professor Bender, first of all, we would like to thank you for accepting this interview. Would you tell us broadly what combinatorics is?

Bender: It is the study of leftover subjects that do not have too much structure and have some aspect of finiteness. What I mean by "leftover" is that combinatorics is the new player on the block and some combinatorial things had been claimed by other fields. For example, partitions of numbers began in number theory and some aspects of algebra are basically combinatorial. Since these leftovers were often considered uninteresting or puzzles (like the seven bridges of Königsberg ${ }^{1}$ ), it took some time for combinatorics to gain respect in the mathematical community. "Too much structure" is not something I will even try to define. So what aspects of these things do combinatorialists study? What comes to mind are enumeration, additional structure (e.g. the size of the largest component in a random graph), existence (e.g. Hamiltonian paths), and construction (e.g. block designs). Speaking of block designs, they are an interesting example of a leftover. Statisticians wanted them to use but not study these leftovers, so combinatorics was able to claim them for study.

Mansour: What do you think about the development of the relations between combinatorics and the rest of mathematics?

Bender: Predictably, combinatorics has made use of tools in the rest of mathematics. But as a late arrival, combinatorics has had a lesser effect on most other areas of mathematics. More interesting to me is the rapid use combinatorics

\footnotetext{
The authors: Released under the CC BY-ND license (International 4.0), Published: March 19, 2021

Toufik Mansour is a professor of mathematics at the University of Haifa, Israel. His email address is tmansour@univ.haifa.ac.il

${ }^{1}$ L. Euler, Solutio problematis ad geometriam situs pertinentis, Comment. Acad. Sci. U. Petrop 8 (1736), 128-40.
} 
has found in other sciences, which is also predictable given that combinatorics deals with finite matters. Don Knuth ${ }^{2}$ is much more able than I to discuss its connections with computer science, so I will ignore that. Ecology, epidemiology, and sociology are among the areas that make use of graph theory. Parts of chemistry and genetics make use of combinatorics. I am sure there are other applications I am not aware of. Since the computational difficulty of combinatorial problems often grows rapidly with size, many of these applications have been made possible by the availability of faster and faster computers.

Mansour: What have been some of the main goals of your research?

Bender: I am primarily a problem solver, which may have grown out of a desire to do well on tests. Of course, I try to see if my approach has a broader application. In fact, sometimes I look at a known result and wonder if there is another way to solve the problem that will lead to more. My earlier research was in various areas and then settled down to be primarily enumeration-perhaps because the more I had done the easier it was to do. Problem-solving influenced my teaching, too. For example, I was bothered that the calculus text I was using at Harvard did not have real applications, so I decided to add some which eventually led to a math modeling course and text at UCSD. Mansour: We would like to ask you about your formative years. What were your early experiences with mathematics? Did that happen under the influence of your family or some other people?

Bender: My earliest mathematics memory was in third grade where long division surprised me because dividing one number by another could go on forever after the decimal point. Other than that, math was easy but not interesting, until I got to algebra in high school. Mathematics could be fun! I finished reading the textbook by Christmas.

Mansour: Were there specific problems that made you first interested in combinatorics?

Bender: My early work was in algebra and some of it had a combinatorial flavor. I found combinatorics interesting but do not recall any specific problems that got me started.
Mansour: How did you get interested in the asymptotic enumeration?

Bender: Jay Goldman and I were teaching a combinatorics course jointly at Harvard and thought we might write a text. I remarked that we should include something about asymptotics based on what Erdős and others had done. He said that since it was my idea, I should learn about it. The book never happened, but my interest did. Admission to candidacy at Caltech is an oral exam after which I was told that I was weak in analysis and must take another course in it. Fortunately, combinatorial asymptotics was in its infancy when I started, so my weak background was not a problem and later I had co-authors who were stronger than I.

Mansour: What was the reason you chose the California Institute of Technology for your Ph.D. and your advisor Olga Taussky-Todd?

Bender: Since I was an Air Force brat, subconsciously I may not have wanted one more move. My conscious rational reason was that I was interested in three areas based on undergraduate courses. I thought I might work with one of those faculty members at Caltech. Here is what happened:

- Elementary number theory with Morgan Ward: Unfortunately, he died of cancer the summer I finished my undergraduate work.

- Lattices with Robert Dilworth: His class dealt primarily with finite lattices and led to my second undergraduate paper. ${ }^{3}$ Sadly for me, his interests had shifted to infinite lattices and I had little interest in them.

- Enumerative combinatorics with Marshall Hall, Jr.: Although his combinatorics class covered various areas, I learned too late that his research interest was design theory.

Fortunately, I found out in my first year as a graduate student that Olga Taussky-Todd's interest in finite matrices fit well with my interests.

Mansour: What was the problem you worked on in your thesis?

Bender: It was in algebra, although parts of it had a combinatorial flavor. The title of my thesis was Symmetric Representation of an Integral Domain over a Subdomain ${ }^{4}$. The representation was by finite matrices. Charac-

\footnotetext{
${ }^{2}$ See http://ecajournal.haifa.ac.il/Volume2021/ECA2021_S3I9.pdf.

${ }^{3}$ Numerical identities in lattices with an application to Dirichlet products. Proc. Amer. Math. Soc. 15 (1964) 8-13.

${ }^{4}$ See https://thesis.library.caltech.edu/9157/.
} 
teristic polynomials and number theory were involved. Some of the work was with $2 \times 2$ matrices over $\mathbb{Z}$.

Mansour: What would guide you in your research? A general theoretical question or a specific problem?

Bender: As mentioned earlier, I'm mostly a problem solver, so I usually started with a fairly specific problem.

Mansour: When you are working on a problem, do you feel that something is true even before you have the proof?

Bender: Yes - but I am not always right! An easily described early example: I was looking at recursions for numbers $a(n+1, k)$ in terms of $a(n, j)$ with nonnegative coefficients depending on $n, k, j$. Based on some common combinatorial numbers I thought that the distribution would tend to a normal as $n \rightarrow \infty$. After getting nowhere I found a simple bimodal counterexample.

Mansour: What three results do you consider the most influential in combinatorics during the last thirty years?

Bender: I don't think specific results were the most influential. The development of faster computers that allowed new applications of combinatorics and consequently spurred development of the field was quite influential. In enumerative combinatorics, I think the books by Erdös and Spencer (Probabilistic Methods in Combinatorics $^{5}$ ) and Flajolet and Sedgewick (Analytic Combinatorics ${ }^{6}$ ) have been influential for people entering the field. I do not feel competent enough to make statements about graph theory or algebraic combinatorics.

Mansour: What are the top three open questions in your list?

Bender: I do not have a list. Of course like a lot of people I would like to know the answer to $P=N P$ and that may involve combinatorics.

Mansour: Do you think that there are core or mainstream areas in mathematics? Are some topics more important than others?

Bender: Yes to both, but things change. Also, while most people might agree on the core areas, they would disagree on the importance of various topics. As a general rule, I would say that the more assumptions that are imposed about what is being studied, the less important the results.

Mansour: What do you think about the distinction between pure and applied mathematics that some people focus on? Is it meaningful at all in your case? How do you see the relationship between so-called "pure" and "applied" mathematics?

Bender: I assume here you are talking about research, not just an application such as using a statistical test to study some data. Given that, I think it is more of a distinction between pure and applied mathematicians - what is the motivation for studying something. For example, if one person studies large random graphs because he is interested in their implications for the internet, and another studies them because he is interested in what the graphs look like, they may do the same work but one is applied and the other is pure.

Mansour: What advice would you give to young people thinking about pursuing a research career in mathematics?

Bender: Do you love it enough? Do you have a talent for it? Job opportunities are not as plentiful as they once were, so what can you do if you do not get a research position?

Mansour: Would you tell us about your interests besides mathematics?

Bender: I enjoy reading fiction and nonfiction. The fiction is mostly mysteries and science fiction. The nonfiction is mostly science, current affairs, and history. Before Covid, I enjoyed going to plays. I enjoy gardening, but I am doing less due to age and some nerve damage in my spine.

Mansour: Pizza slicing, phi's, and the Riemann hypothesis ${ }^{7}$ ? It is an interesting title of one of your papers, co-authored with Oren Patashnik and Howard Rumsey Jr. How does the Riemann Hypothesis enter the picture? What was the motivation for this paper?

Bender: The paper is partially expository. My recollection is that it was written to entertain and educate students. It deals with the number of regions a unit square is divided into by certain lines with integral slopes. (Yes a

\footnotetext{
${ }^{5}$ P. Erdős and J. Spencer, Probabilistic methods in combinatorics, Probability and Mathematical Statistics, Volume 17, Academic Press [A subsidiary of Harcourt Brace Jovanovich, Publishers], New York-London, 1974.

${ }^{6}$ P. Flajolet and R. Sedgewick, Analytic combinatorics, Cambridge University Press, Cambridge, 2009.

${ }^{7}$ E. A. Bender, O. Patashnik, and H. Jr. Rumsey, Pizza slicing, phi's, and the Riemann hypothesis, Amer. Math. Monthly 101:4 (1994), 307-317.
} 
square pizza, no $\pi$ here.) As I recall, the title and much of the writing were due to Oren who may have come up with the question. The count depends on the sum of the Euler phi function and the accuracy of asymptotics for that depends on the Riemann hypothesis.

Mansour: There is a nice formula ${ }^{8}$ on your home page. Would you tell us about this formula and why you chose it specifically for your web page? Which formulas from combinatorics are the top three for you?

Bender: I chose it because it is a simple question with a fairly simple nonintuitive formula and the paper is expository. So I thought it could be a way of getting someone interested in enumerative combinatorics. I do not have any favorite formulas I just take them as they are. If I had to choose a formula for a T-shirt. It would not be from combinatorics. It would be $e^{i \pi}+1=0$ because of the amount of mathematical understanding involved from simple arithmetic to a sophisticated understanding of basic constants and exponentiation.

Mansour: In your work, you have extensively used combinatorial reasoning to address important problems. How do enumerative techniques engage in your research?

Bender: I am not sure I understand you clearly. Much of my research was in the enumeration. (Yes, "was" not "is". I have not been doing research since I do not think I am up to doing the level of research I would want.)

Mansour: You have also published some papers in biology journals. How were you driven to work on such aspects of mathematical biology? Would you tell us about some questions from biology which could take the attention of combinatorialists?

Bender: Because some ecology graduate students took my math modeling course, I met the ecology professors Ted Case and Mike Gilpin. Our joint work was forty years ago and is not combinatorial, so I can not make any recommendations of combinatorial problems. Anyone who is interested should talk to someone in biology perhaps about gene sequencing, ecology, or epidemiology.

Mansour: On your home page, in a few lines, you have explained "Why do We Need Proofs?" What do you think of computer- assisted proofs?

Bender: Suppose a person gives a relatively short proof. If someone reliable looks at it and says it is fine, I think it is reasonable to accept it. But if the proof is very long or intricate, I would like more than one person to look at it. The same thing applies to computer assisted proof, but now we have to consider more: the method, the program, the compiler, and maybe the computer. So, after checking the method, we might want someone else to program it in another language.

Mansour: You have some notes regarding Health/Nutrition on your web page. What was the reason you have decided to do this? I have been experiencing a complex problem at home recently. One of my daughters, Atil, has decided to follow a vegetarian diet based on some ethical issues. Whenever she wants to discuss it, I try to escape by saying that "Ohh, this is a challenging real-life problem; I am a mathematician, let me finish this paper, later we can talk about it!" But I have no satisfactory answer! What do you think about eating meat not as a nutritional issue but as a philosophical issue?

Bender: A while ago I was collecting information for use by my wife and me. Then I thought someone else might be interested in it so I put it on my web page. Of course, it is out of date, not just because of new information but also because a large fraction of results change when people attempt to replicate studies. Some animals, including humans, eat other animals - it is the way the world is. So I have no problem with the idea of eating meat. I do have a problem with how most animals are treated to produce meat or milk or eggs as cheaply as possible. Most of the time in the grocery store I pay attention to that. I think a side benefit is that more humanely produced products are healthier.

Mansour: In a very recent short article, published at Newsletter of the European Mathematical Society, professor Melvyn B. Nathanson $^{9}$, while elaborating on the ethical aspects of the question "Who Owns the Theorem?" concluded that "Mathematical truths exist, and mathematicians only discover them." On the other side, there are opin-

\footnotetext{
${ }^{8}$ See http://www.math.ucsd.edu/ ebender/reprints/pubs.html.

${ }^{9}$ See https ://www.ems-ph.org/journals/newsletter/pdf/2020-12-118.pdf.
} 
ions around that "mathematical truths are invented". As a third way, some people claim that it is both invented and discovered? What do you think about this old discussion?

Bender: I have not seen the article. The question sounds simple, but there are some twists. What are the rules of the game? Do we allow or deny the continuum hypothesis? Are we limited to constructible real numbers? Et cetera. So the rules of the game are invented. We might then conclude that, given the rules, the truths are discovered. But Gödel's Incompleteness Theorem ${ }^{10}$ muddies the waters if we confound the ideas of truth and provability within a given set of rules. Is it okay to say something is true if we know it is but can not prove it with the set of rules we are using? My research topics allowed me to solve these problems by ignoring them.

Mansour: Would you tell us about your thought process for the proof of one of your favorite results? How did you become interested in that problem? How long did it take you to figure out a proof? Did you have a "eureka moment"?

Bender: I am assuming "favorite" does not mean "important". In that case, I might pick an early result - it is like a first love. But I do not remember the details and they are not combinatorics. I know that I had a "eureka moment" when I was working on the asymptotic enumeration of rooted graphs on surfaces - basically how much various terms in the recursion mattered, but again I forget the details. So I will tell you about a simple problem that I enjoyed. A composition $c_{1}, c_{2}, \cdots$ of $n$ is called Carlitz ${ }^{11}$ if $c_{k} \neq c_{k+1}$ for all $k$. They were studied by setting up a recursion based on adding one more part. It took a little work to show that the circle of convergence had a single, simple pole. All this was done and published by someone. I wondered "What about different restrictions?" The "eureka moment" came fairly quickly: Instead of adding an additional part, add one to each part. This meant I could study things like $c_{k}-c_{k+1} \notin \mathcal{S}$ for some set $\mathcal{S}$, and the nature of the singularity was easy to see.

Mansour: Is there a specific problem you have been working on for many years? What progress have you made?

Bender: Here is a simple problem that I thought about off and on for a while with no real progress. Call the composition $c_{1}, c_{2}, \cdots$ of $n d$-Carlitz if $c_{k} \neq c_{k+i}$ for all $k$ and $1 \leq i \leq d$. So Carlitz compositions are 1-Carlitz. I can not even say much about 2-Carlitz compositions.

Mansour: Some entrepreneurs and scientists have very ambitious plans to go to Mars. Many people criticize such endeavors by proposing that the money needed for this project could immediately help to fight against diseases, poverty, climate change and instead support other practical scientific projects. What is your point of view on going to Mars?

Bender: I look at it from the point of view of the benefits of going to the moon. Here is what I see.

- It led to the development of technology useful here. I think that is less likely with going to Mars.

- It got people interested in doing things in space. That interest has waned and we need interest if space exploration is going to be funded. I simply do not know if planning a trip to Mars will help that much.

- It enhanced U.S. prestige in the cold war. Does that matter so much now?

- The rock samples brought back led to knowledge. The same would be true of going to Mars, but do we need people there? There has been robotic retrieval of samples from the moon. NASA's first phase of robotic retrieval recently landed on Mars. The argument can be made that a scientist on Mars could make a better choice. On the other hand, robotics keeps improving and humans on Mars have built-in limitations. Besides, working on improving robotics will be useful in exploring beyond Mars.

Thus, cost aside, I think sending people to Mars may be the wrong approach.

Mansour: My last question is philosophical: have you figured out why we are here?

Bender: If "why" means the purpose, then I think the question makes an assumption I do not accept. If "why" is more along the lines of "what makes life satisfying", then that is up to the individual to decide. If it is a scientific

\footnotetext{
${ }^{10}$ For example, see the entry by Panu Raatikainen in the Stanford Encyclopedia of Philosophy, 2013.

${ }^{11}$ L. Carlitz, Restricted compositions, The Fibonacci Quart. 14 (1976), 254-264.
} 
question, evolution takes care of the biological part of it, but physics has not explained why our universe exists. There is a joke about that whose source I do not know:

- Suppose nothing exists.

- There would be no rules since rules are something.

- If there are no rules, then nothing is impossible.
- This contradicts the assumption that nothing exists.

- So something must exist and our universe happens to be at least part of it.

Mansour: Professor Edward A. Bender, I would like to thank you for this very interesting interview on behalf of the journal Enumerative Combinatorics and Applications. 